\title{
BMJ Open Constipation and risk of cardiovascular diseases: a Danish population-based matched cohort study
}

\author{
Jens Sundbøll (D) , ${ }^{1}$ Szimonetta Komjáthiné Szépligeti, ${ }^{1}$ Kasper Adelborg (iD , ${ }^{1}$ \\ Péter Szentkúti, ${ }^{1}$ Hans Gregersen, ${ }^{2}$ Henrik Toft Sørensen ${ }^{1}$
}

To cite: Sundbøll J, Szépligeti SK, Adelborg K, et al. Constipation and risk of cardiovascular diseases: a Danish population-based matched cohort study. BMJ Open 2020;10:e037080. doi:10.1136/ bmjopen-2020-037080

- Prepublication history and additional material for this paper are available online. To view these files, please visit the journal online (http://dx.doi. org/10.1136/bmjopen-2020037080).

Received 17 January 2020 Revised 23 May 2020 Accepted 23 June 2020

Check for updates

(c) Author(s) (or their employer(s)) 2020. Re-use permitted under CC BY-NC. No commercial re-use. See rights and permissions. Published by BMJ.

${ }^{1}$ Department of Clinical Epidemiology, Aarhus University Hospital, Aarhus, Denmark

${ }^{2}$ GIOME, Department of Surgery, Chinese University of Hong Kong, Shatin, Hong Kong

Correspondence to Dr Jens Sundbøll; jens.sundboll@clin.au.dk

\section{ABSTRACT}

Objectives To assess the risks of myocardial infarction, stroke, peripheral artery disease, venous thromboembolism, atrial fibrillation or atrial flutter and heart failure in patients with constipation compared with a general population cohort.

Design Population-based matched cohort study. Setting All Danish hospitals and hospital outpatient clinics from 2004 to 2013.

Participants Patients with a constipation diagnosis matched on age, sex and calendar year to 10 individuals without constipation from the general population. Main outcomes measures Comorbidity-adjusted and medication-adjusted hazard ratios (aHRs) for cardiovascular outcomes based on Cox regression analysis.

Results 83239 patients with constipation were matched to 832384 individuals without constipation. The median age at constipation diagnosis was $46.5 \%$ and $41 \%$ were men. Constipation was strongly associated with venous thromboembolism (aHR 2.04, 95\% Cl 1.89 to 2.20), especially splanchnic venous thrombosis $(4.23,95 \% \mathrm{Cl}$ 2.45 to 7.31 ). Constipation was also associated with arterial events, including myocardial infarction (1.24, $95 \% \mathrm{Cl} 1.14$ to 1.35$)$, ischaemic stroke $(1.50,95 \% \mathrm{Cl} 1.41$ to 1.60$)$, haemorrhagic stroke $(1.46,95 \% \mathrm{Cl} 1.26$ to 1.69$)$, peripheral artery disease $(1.34,95 \% \mathrm{Cl} 1.20$ to 1.50$)$, atrial fibrillation or atrial flutter $(1.27,95 \% \mathrm{Cl} 1.20$ to 1.34$)$ and heart failure $(1.52,95 \% \mathrm{Cl} 1.42$ to 1.62$)$. The associations were strongest during the first year after the constipation diagnosis and strengthened with an increased number of laxative prescriptions.

Conclusions Constipation was associated with an increased risk of several cardiovascular diseases, in particular venous thromboembolism.

\section{INTRODUCTION}

Constipation causes substantial morbidity worldwide $^{12}$ and is one of the most prevalent conditions presenting to general practitioners, medical specialists and surgeons across subspecialties. ${ }^{3}$ The prevalence of constipation ranges from $3 \%$ to $79 \%$ in various adult populations depending on age, sex and the definition of constipation. ${ }^{2}$ 4-9 Among patients hospitalised for

\section{Strengths and limitations of this study}

- The population-based design within the setting of a tax-supported universal healthcare system limited selection bias.

- We only included constipated patients in contact with the healthcare system, which may limit the generalisability.

- The positive predictive value of the constipation diagnosis in the Danish National Patient Registry has not been examined in detail, but was confirmed by the treating physician and therefore assumed high.

- Cardiovascular diagnoses generally have very high positive predictive values in the Danish National Patient Registry.

- Despite the use of several approaches to control for and examine the potential impact of confounding, unmeasured confounding cannot be entirely excluded.

cardiovascular disease, the prevalence of constipation is approximately $50 \% .^{10}$

Constipation and cardiovascular diseases share common risk factors, including age, ${ }^{8}$ use of non-aspirin non-steroidal antiinflammatory drugs, ${ }^{11}$ diabetes mellitus, depression, ${ }^{12}$ lack of physical exercise ${ }^{13}$ and low dietary fibre intake. ${ }^{13}$ However, a substantial proportion of cardiovascular diseases cannot be explained by traditional cardiovascular risk factors. ${ }^{14}$ Constipation may be a risk factor for cardiovascular diseases via several putative mechanisms. Disturbances in the gut microbiome are common in patients with constipation ${ }^{15}$ and have been associated with arterial stiffness, ${ }^{16}$ increased blood pressure ${ }^{17}$ atherosclerotic cardiovascular diseases ${ }^{18-20}$ and heart failure. ${ }^{21}$ However, the associations have not been investigated specifically in constipated patients with dysbiosis. In addition, strain at stool and associated mental stress increase the blood pressure ${ }^{10}$ which is a risk factor for atherosclerotic, haemorrhagic and arrhythmic cardiovascular diseases. ${ }^{22} 23$ 
Information on the association between constipation and cardiovascular disease is currently limited. Two studies have examined this association, both focusing only on atherosclerotic cardiovascular disease and relying on self-reported constipation. In a cohort of postmenopausal women, only severe constipation was associated with a 1.2-fold increased risk of atherosclerotic cardiovascular disease. ${ }^{24}$ In a cohort of US veterans, constipation was associated with a 1.1-fold increased risk of coronary heart disease and a 1.2-fold increased risk of ischaemic stroke. $^{25}$

Considering the sheer number of patients with constipation, ${ }^{3}$ any association with cardiovascular disease would have public health interest. Therefore, we conducted a large population-based cohort study of patients with a first-time diagnosis of constipation to examine the subsequent risk of common cardiovascular diseases compared with risks in a matched general population cohort.

\section{METHODS}

\section{Setting and design}

We conducted this population-based cohort study in Denmark, which had a cumulative population of 6482126 inhabitants during the study period. The Danish National Health Service provides universal tax-supported healthcare, guaranteeing unfettered access to general practitioners and hospitals. ${ }^{26}$ Accurate and unambiguous linkage of all registries in Denmark is possible at the individual level using the unique central personal registry number assigned to each Danish citizen at birth and to residents on immigration. ${ }^{27}$

\section{Patients with constipation}

Selection of patients is presented in the study flowchart (figure 1). We used the Danish National Patient Registry $(\mathrm{DNPR})^{28}$ to identify all patients with a first-time inpatient or outpatient hospital diagnosis of constipation during the study period (1 July 2004 to 30 November 2013). The DNPR has recorded information on dates of admission and discharge from all Danish non-psychiatric hospitals since 1977 and from emergency room and outpatient clinic visits since $1995 .{ }^{28}$ Each hospital discharge or outpatient clinic visit is recorded with one primary diagnosis (ie, the main reason for admission) and one or more secondary diagnoses classified according to the International Classification of Diseases, Eighth Revision (ICD-8) through 1993 and 10th Revision (ICD-10) thereafter. ${ }^{28}$ We identified patients with constipation using both the primary and secondary inpatient and outpatient diagnoses. We excluded patients with a previous or concurrent inpatient or outpatient diagnosis of any of the study outcomes (myocardial infarction, ischaemic or haemorrhagic stroke, peripheral artery disease, venous thromboembolism, atrial fibrillation or atrial flutter and heart failure). The index date was defined as the date of first hospital admission with constipation (for inpatients) or the date of the first hospital clinic visit with a constipation constipation diagnosis, Denmark, 2004-2013 $\mathrm{N}=106,997$

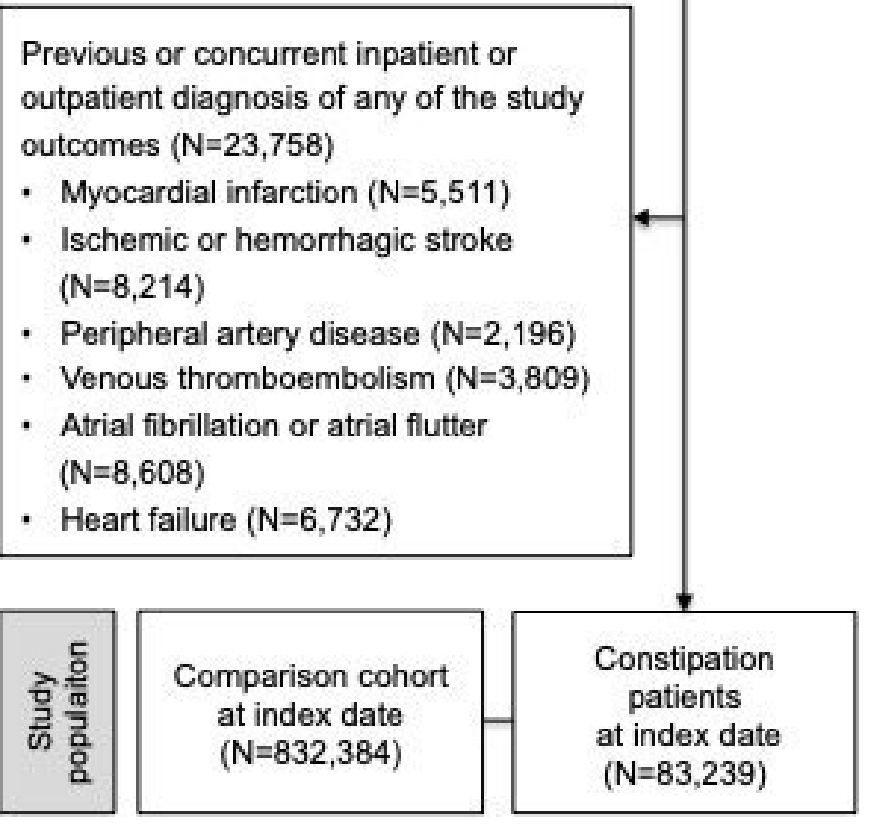

Figure 1 Study flowchart. Because several patients had more than one previous or concurrent diagnosis of the study outcomes, the sum of these exceeds the number of patients excluded (23 758).

diagnosis (for outpatients). The ICD and Anatomical Therapeutic Chemical (ATC) codes used in this study are provided in online supplementary table S1.

Many patients with constipation are managed solely in primary care and not captured in hospital-based registries. Therefore, we performed a separate analysis in which we redefined the constipation cohort to also include prescriptions for laxatives. In this cohort including patients from general practice, we defined the constipation index date as the date of a hospital diagnosis or the date of a second prescription for laxatives, whichever occurred first. A second prescription was required to ensure that constipation was ongoing.

To evaluate the impact of constipation severity on the risk of study outcomes, we categorised patients based on the number of prescriptions for laxatives redeemed from 1 year prior to 3 months after the constipation diagnosis: low-intensity use corresponding to $0-1$ prescriptions and high-intensity use corresponding to $\geq 2$ prescriptions. For this purpose, we used data from the Danish National Health Service Prescription Database established in $2004 .^{29}$ For each prescription redeemed after 2004, the patient's central personal registry number, the amount and type of drug prescribed according to the ATC classification system and the date the drug was dispensed have been transferred electronically from community pharmacies to the Prescription Database. To avoid conditioning 
on the future, the index date in this analysis was changed to 3 months after the constipation diagnosis or the matching date for individuals in the general population comparison cohort. ${ }^{30}$

\section{General population comparison cohort}

To put constipation into a population context, we created a comparison cohort from the general population using the Danish Civil Registration System. This system has recorded all changes in vital status and migration for the entire Danish population since 1968, with daily electronic updates. ${ }^{27}$ For each patient in the constipation cohort, up to 10 individuals from the general population were randomly selected from the Civil Registration System and matched on sex and single year of age during the calendar year of the patient's constipation diagnosis. We selected 10 individuals for each constipation patient, as this approach would not be associated with extra expense and helped ensure adequate precision of our estimates, including in subcohorts. We used matching with replacement (ie, individuals from the general population comparison cohort could be matched with more than one patient with constipation). ${ }^{31}$ Each member of the comparison cohort was assigned an index date corresponding to the date of admission or outpatient visit for the corresponding patient with constipation. All members of the comparison cohort were required to be alive on the date the corresponding patient was diagnosed with constipation. We included only comparison cohort members with no previous hospital-based diagnosis of the study outcomes or previous constipation diagnoses. Individuals from the comparison cohort who were diagnosed with constipation during follow-up were transferred to the constipation cohort at that point and follow-up was discontinued in the comparison cohort.

\section{Cardiovascular outcomes}

The outcomes were specified a priori according to hypotheses proposed in the introduction and included first-time myocardial infarction, ischaemic or haemorrhagic stroke, peripheral artery disease, venous thromboembolism, atrial fibrillation or atrial flutter and heart failure. These conditions were ascertained using all available primary and secondary inpatient and outpatient diagnoses recorded in the DNPR. We separately analysed the anatomical locations of venous thromboembolisms (ie, deep venous thrombosis, pulmonary embolism or splanchnic venous thrombosis). To explore underlying mechanisms, we assessed the risk of unprovoked and provoked venous thromboembolism; provoked was defined on the basis of a diagnosis of malignancy any time before the venous thromboembolism, or pregnancy, fracture/trauma or surgery within 90 days before the venous thromboembolism (online supplementary table S2) ${ }^{32}$ Registration of cardiovascular diagnoses in the DNPR is accurate, with validation studies consistently reporting positive predictive values $>80 \%$ for most conditions. ${ }^{33}$ As approximately two-thirds of all unspecified strokes are known to be ischaemic strokes,${ }^{34}$ we classified unspecified strokes as ischaemic strokes.

\section{Covariables}

Using the full hospital history (inpatient and outpatient diagnoses) recorded in the DNPR before the index date, we obtained information on the following potential constipation-related conditions: ${ }^{12}$ hypothyroidism, hyperthyroidism, pregnancy within 90 days, depression, Parkinson's disease, multiple sclerosis, colon, rectal and anal cancer, other gastrointestinal cancers, Crohn's disease, ulcerative colitis and paralytic ileus. In addition, we collected data on several cardiovascular risk factors: chronic pulmonary disease (as a measure of chronic smoking exposure), valvular heart disease, diabetes mellitus, hypertension, hypercholesterolemia, obesity, chronic kidney disease, liver disease and alcoholismrelated disorders. We also retrieved information on medication use within 90 days preceding the index date from the Danish National Health Service Prescription Database,${ }^{29}$ including medications that can induce constipation $^{12}$ (iron supplements, opioids, calcium channel blockers, anticholinergic drugs, dopaminergic drugs, tricyclic antidepressants, diuretics, aspirin and nonsteroidal anti-inflammatory drugs) and cardiovascular drugs (vitamin $\mathrm{K}$ antagonists, direct oral anticoagulants, angiotensin-converting enzyme inhibitors, angiotensin receptor blockers, beta-blockers, P2Y12 inhibitors, digoxin, amiodarone, nitrates and statins).

\section{Statistical analysis}

We tabulated the distributions of the covariables for the constipation cohort and the general population cohort for comparison. We followed both cohorts from the index dates until the date of myocardial infarction, ischaemic stroke, haemorrhagic stroke, peripheral artery disease, venous thromboembolism, atrial fibrillation or atrial flutter, heart failure, emigration, death or end of follow-up (30 November 2013). After an initial event, we continued to follow patients for subsequent cardiovascular events to avoid informative censoring and to understand the full spectrum and extent of cardiovascular morbidity associated with constipation. We calculated and graphically illustrated the cumulative incidence per 1000 persons for each outcome for $0-1$ year, $>1-5$ years, $>5-10$ years and $0-10$ years, accounting for the competing risk of death. ${ }^{35}$ We then used matching factor-stratified (conditional) Cox proportional hazards regression analysis ${ }^{36}$ to compute hazard ratios adjusted (aHRs) for the categorical variables listed in table 1. To examine potential disparities in risk in subgroups of patients, the results were stratified by sex, age group, constipation-related conditions and drugs, type of hospital contact (inpatient/outpatient), type of diagnosis (primary/secondary) and number of cardiovascular risk factors $(0,1, \geq 2)$. Using log-log plots, we examined potential deviations from the proportionality of hazard assumption in the analysed follow-up periods. We found no violations of the assumption. 
Table 1 Characteristics of patients with constipation and members of the general population comparison cohort

\section{Constipation cohort, $\mathrm{n}=83239$}

\begin{tabular}{|c|c|c|}
\hline Median age, years (25th-75th percentile) & $46.5(8.6-69.8)$ & $46.5(8.6-69.8)$ \\
\hline \multicolumn{3}{|l|}{ Age groups, years } \\
\hline$<55$ & $47645(57.2)$ & $476547(57.3)$ \\
\hline $55-64$ & $9440(11.3)$ & $94572(11.4)$ \\
\hline $65-74$ & $10877(13.1)$ & $108741(13.1)$ \\
\hline$\geq 75$ & $15277(18.4)$ & $152524(18.3)$ \\
\hline Male & $34138(41.0)$ & $341374(41.0)$ \\
\hline \multicolumn{3}{|c|}{ Year of constipation diagnosis/index date } \\
\hline $2004-2008$ & $30751(36.9)$ & $307510(36.9)$ \\
\hline $2009-2013$ & $52488(63.1)$ & $524874(63.1)$ \\
\hline \multicolumn{3}{|l|}{ Type of constipation diagnosis } \\
\hline Primary diagnosis & $54074(65.0)$ & - \\
\hline Secondary diagnosis & $29165(35.0)$ & - \\
\hline \multicolumn{3}{|l|}{ Type of hospital contact for constipation } \\
\hline Inpatient & $44851(53.9)$ & - \\
\hline Outpatient & $38388(46.1)$ & - \\
\hline \multicolumn{3}{|l|}{ Constipation-related conditions } \\
\hline Hypothyroidism & $1226(1.5)$ & $6167(0.7)$ \\
\hline Hyperthyroidism & $1244(1.5)$ & $9463(1.1)$ \\
\hline $\begin{array}{l}\text { Pregnancy within } 90 \text { days of the index } \\
\text { date }\end{array}$ & $678(0.8)$ & $2629(0.3)$ \\
\hline Depression & $2237(2.7)$ & $6921(0.8)$ \\
\hline Parkinson's disease & $644(0.8)$ & $1856(0.2)$ \\
\hline Multiple sclerosis & $588(0.7)$ & $1477(0.2)$ \\
\hline Colon, rectal and anal cancer & $3459(4.2)$ & $7005(0.8)$ \\
\hline Other gastrointestinal cancers & $1901(2.3)$ & $1037(0.1)$ \\
\hline $\begin{array}{l}\text { Crohn's disease, ulcerative colitis, } \\
\text { paralytic ileus }\end{array}$ & $3873(4.7)$ & $8892(1.1)$ \\
\hline \multicolumn{3}{|l|}{ Cardiovascular risk factors } \\
\hline Chronic pulmonary disease & $23204(27.9)$ & $163581(19.7)$ \\
\hline Valvular heart disease & $946(1.1)$ & $6590(0.8)$ \\
\hline Diabetes mellitus & $5366(6.4)$ & $33563(4.0)$ \\
\hline Hypertension & $8929(10.7)$ & $54132(6.5)$ \\
\hline Hypercholesterolemia & $1826(2.2)$ & $10972(1.3)$ \\
\hline Obesity & $3340(4.0)$ & $17399(2.1)$ \\
\hline Chronic kidney disease & $1220(1.5)$ & $5062(0.6)$ \\
\hline Liver disease & $1308(1.6)$ & $4428(0.5)$ \\
\hline Alcoholism-related disorders & $2776(3.3)$ & $10832(1.3)$ \\
\hline \multicolumn{3}{|l|}{ Drugs associated with constipation* } \\
\hline Iron supplements & $128(0.2)$ & $87(0.0)$ \\
\hline Opioids & $17172(20.6)$ & $30089(3.6)$ \\
\hline Calcium channel blockers & $5445(6.5)$ & 49499 (5.9) \\
\hline Anticholinergic drugs & $366(0.4)$ & $998(0.1)$ \\
\hline Dopaminergic drugs & $1041(1.3)$ & $4064(0.5)$ \\
\hline Tricyclic antidepressants & $2328(2.8)$ & $5471(0.7)$ \\
\hline
\end{tabular}

Continued 
Table 1 Continued

\begin{tabular}{lcr}
\hline & Constipation cohort, $\mathbf{n = 8 3 2 3 9}$ & Comparison cohort, $\mathbf{n = 8 3 2 3 8 4}$ \\
\hline Diuretics & $9967(12.0)$ & $69706(8.4)$ \\
Aspirin & $6000(7.2)$ & $49569(6.0)$ \\
Non-steroidal anti-inflammatory drugs & $10154(12.2)$ & $52753(6.3)$ \\
\hline $\begin{array}{l}\text { Cardiovascular drugs* } \\
\text { Vitamin K antagonists and direct oral }\end{array}$ & $298(0.3)$ & $2637(0.3)$ \\
anticoagulants & & \\
Angiotensin-converting enzyme & $8078(9.7)$ & $81854(9.8)$ \\
inhibitors and angiotensin receptor & & $38630(4.6)$ \\
blockers & $3854(4.6)$ & $2019(0.2)$ \\
Beta-blockers & $353(0.4)$ & $2800(0.3)$ \\
P2Y12 inhibitors & $293(0.4)$ & $52(0.0)$ \\
Digoxin & $22(0.0)$ & $5477(0.7)$ \\
Amiodarone & $978(1.2)$ & $53432(6.4)$ \\
\hline Nitrates & $5549(6.7)$ & \\
Statins & & \\
\hline
\end{tabular}

Data are reported as $\mathrm{n}(\%)$ unless otherwise specified.

${ }^{*}$ Redeemed prescription within $\leq 90$ days before the index date.

\section{Sensitivity analyses}

We performed several sensitivity analyses to test the robustness of our study findings. First, to improve the specificity of the stroke diagnosis, we implemented a stricter definition of stroke, requiring a CT or MRI scan of the brain in the record of the incident hospital contact for stroke. Second, because 'unspecified stroke' diagnoses were classified in the main analysis as ischaemic strokes, as the most likely subtype ${ }^{34}$ we repeated the analysis with separate assessments of specified ischaemic stroke and unspecified stroke to test the validity of this assumption. Third, as a bias analysis, we calculated E-values for estimates of myocardial infarction and venous thromboembolism as representative outcomes and the corresponding lower limit of the $95 \%$ CI during $0-10$ years of follow-up. The E-value is the minimum strength of the confounder association with both exposure and outcome that must be present, above and beyond the measured covariates, for an unmeasured confounder to explain away an association. This allowed us to assess how strong an unmeasured confounder (eg, immobilisation) would have to be to explain away the observed exposure-outcome association. ${ }^{37}$

All statistical analyses were performed using SAS V.9.4 (SAS Institute, Cary, North Carolina, USA). The data, analytic methods and study materials will not be made available to other researchers for purposes of reproducing the results or replicating the procedure. Such disclosure would conflict with the regulations for use of Danish healthcare data.

\section{Patient and public involvement}

Patients or the public were not involved in the design, or conduct, or reporting, or dissemination plans of our research.

\section{RESULTS}

The study included 83239 patients in the constipation cohort and 832384 individuals in the matched general population comparison cohort. Median age at constipation diagnosis was 46.5 (IQR 8.6-69.8) years, and $41 \%$ of the study population were men. The constipation cohort had an expected higher prevalence of constipationrelated conditions and associated drug use and a slightly higher prevalence of cardiovascular risk factors and associated drug use compared with the general population comparison cohort (table 1).

\section{Constipation and cardiovascular disease risk}

The cumulative incidence per 1000 persons after 10 years of follow-up in the constipation cohort compared with the general population cohort was 17 vs 20 for myocardial infarction, 30 vs 31 for ischaemic stroke, 6 vs 6 for haemorrhagic stroke, 15 vs 10 for peripheral artery disease, 21 vs 15 for venous thromboembolism, 42 vs 54 for atrial fibrillation or atrial flutter and 30 vs 30 for heart failure (figure 2). The cumulative incidence of myocardial infarction, ischaemic stroke and atrial fibrillation or atrial flutter was lower in the constipation cohort because of the competing risk of death.

After adjusting for the covariables, constipation was associated with a 1.2-2-fold increased risk of cardiovascular disease during 0-10 years of follow-up (myocardial infarction: aHR 1.24, 95\% CI 1.14 to 1.35 ; ischaemic stroke: aHR $1.50,95 \%$ CI 1.41 to 1.60 ; haemorrhagic stroke: aHR 1.46, $95 \%$ CI 1.26 to 1.69 ; peripheral artery disease: aHR 1.34, 95\% CI 1.20 to 1.50 ; venous thromboembolism: aHR 2.04, 95\% CI 1.89 to 2.20; atrial fibrillation or atrial flutter: aHR 1.27, 95\% CI 1.20 to 1.34 ; heart failure: aHR 1.52, 95\% CI 1.42 to 1.62; figure 3 ). The risks 
Myocardial infarction

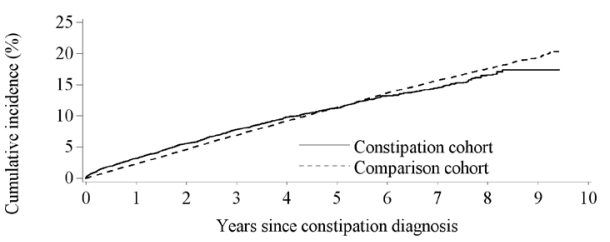

Hemorrhagic stroke

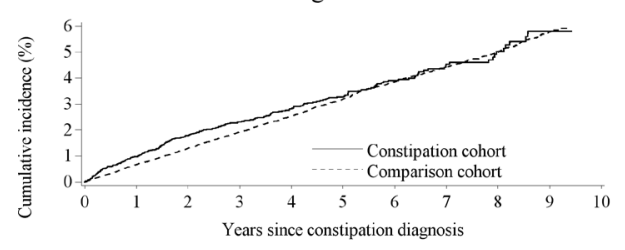

Venous thromboembolism

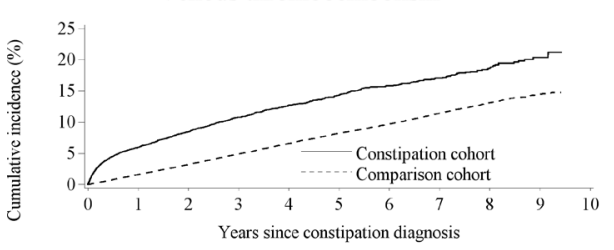

Heart failure

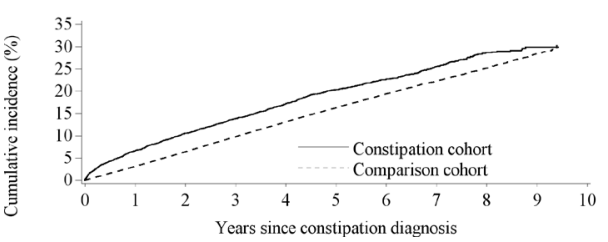

Ischaemic stroke

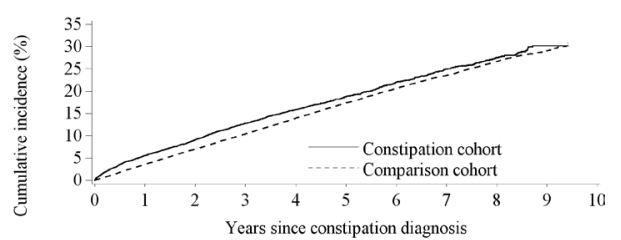

Peripheral artery disease

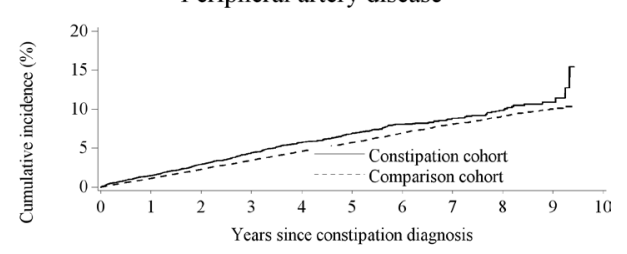

Atrial fibrillation

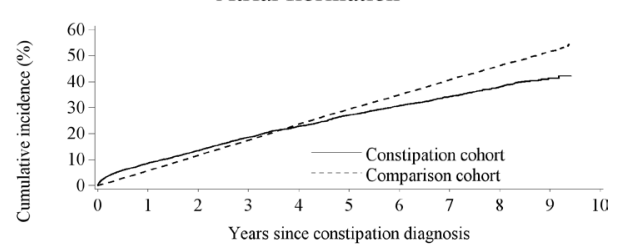

Figure 2 Cumulative risk (\%) of myocardial infarction, ischaemic and haemorrhagic stroke, peripheral artery disease, venous thromboembolism, atrial fibrillation or atrial flutter and heart failure among patients with constipation and members of the general population comparison cohort.

were similar for deep venous thrombosis and pulmonary embolism but markedly higher for splanchnic venous thrombosis (aHR 4.23, 95\% CI 2.45 to 7.31). The risks were only slightly higher for provoked venous thromboembolism than for unprovoked venous thromboembolism (online supplementary table S3).

The excess risk of cardiovascular disease was highest during the first year following the diagnosis of constipation, with a 1.5-fold increased risk of myocardial infarction, an approximately 1.7 -fold increased risk of ischaemic stroke, haemorrhagic stroke and atrial fibrillation or atrial flutter and a 1.2-fold increased risk of peripheral artery disease. The risk was even higher for venous thromboembolism (3.5-fold) and heart failure (2.2-fold). During $>1-5$ years of follow-up, constipation was persistently associated with myocardial infarction (1.2-fold increased risk), ischaemic and haemorrhagic stroke (1.4-fold increased risk), peripheral artery disease and venous thromboembolism (1.5-fold increased risk), heart failure (1.3-fold increased risk) and marginally with atrial fibrillation or atrial flutter (1.1-fold increased risk). During $>5-10$ years of follow-up, constipation was associated primarily with ischaemic stroke (1.5-fold increased risk) and venous thromboembolism (1.3-fold increased risk) and marginally with haemorrhagic stroke and heart failure (figure 3).

When we included prescriptions for laxatives in the definition of constipation, the constipation cohort increased to 109915 patients, $24 \%$ of whom were identified through prescription redemption. Using this expanded definition of constipation did not change the associations for any outcomes (table 2).

\section{Subgroup analyses}

In analyses stratified by the intensity of laxative use before and immediately after the constipation diagnosis, we observed stronger associations with a higher intensity of laxative use for all outcomes, except for peripheral artery disease (table 3 ). The association was particularly strengthened in patients with high-intensity versus lowintensity use for the outcomes ischaemic stroke (3.2-fold and 1.4-fold, respectively) and venous thromboembolism (5.2-fold and 1.7-fold, respectively). The associations were similar among women and men (online supplementary table S4) and generally attenuated with increasing age 


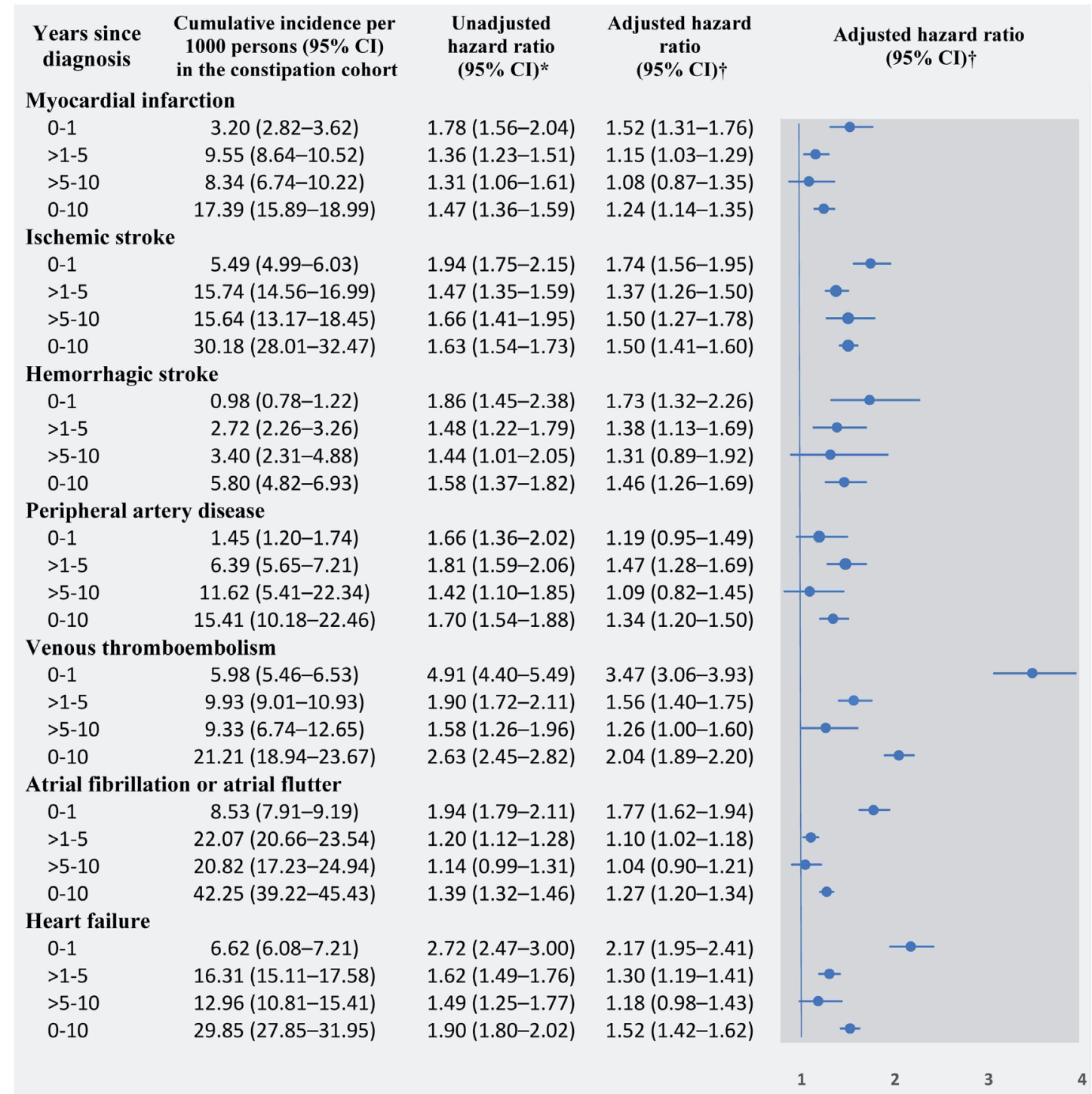

Figure 3 Risk of cardiovascular events among patients with constipation relative to the general population cohort. *Controlled for matching factors (age, sex and calendar year) by study design. †Controlled for matching factors (age, sex and calendar year) by study design and adjusted for hypothyroidism, hyperthyroidism, pregnancy within 90 days before the index date, depression, Parkinson's disease, multiple sclerosis, colon, rectal and anal cancer, other gastrointestinal cancers, Crohn's disease, ulcerative colitis, paralytic ileus, chronic pulmonary disease, valvular heart disease, diabetes mellitus, hypertension, hypercholesterolemia, obesity, chronic kidney disease, liver disease, alcoholism-related disorders, medications associated with constipation (iron supplements, opioids, calcium channel blockers, anticholinergic drugs, dopaminergic drugs, tricyclic antidepressants, diuretics, aspirin and non-steroidal anti-inflammatory drugs) and cardiovascular drugs (vitamin K antagonists, direct oral anticoagulants, angiotensin-converting enzyme inhibitors, angiotensin receptor blockers, beta-blockers, P2Y12 inhibitors, digoxin, amiodarone, nitrates and statins).

(online supplementary table S5). With the exception of haemorrhagic stroke, the associations were stronger in patients with inpatient diagnoses of constipation than in patients with outpatient diagnoses (online supplementary table S6). When restricting to patients with a primary diagnosis of constipation, the associations remained, though marginally attenuated compared with patients with secondary diagnoses of constipation (online supplementary table S7). The associations were slightly weakened in patients diagnosed with constipationrelated conditions or who used drugs that can cause constipation (online supplementary table S8). However, the risks of myocardial infarction, ischaemic stroke and haemorrhagic stroke were higher in patients with constipation who used non-steroidal anti-inflammatory drugs. When restricting the analysis to patients with no cardiovascular risk factors at baseline, the results remained largely unchanged. Only the risk of myocardial infarction increased with an increasing burden of cardiovascular risk factors at baseline (online supplementary table S9).

\section{Sensitivity analyses}

In separate analyses of unspecified stroke and specified ischaemic stroke, the associations were moderately 
Table 2 Risk of cardiovascular events among patients with constipation relative to the general population cohort, redefining constipation as a hospital diagnosis or $\geq 2$ prescriptions for laxatives, whichever came first

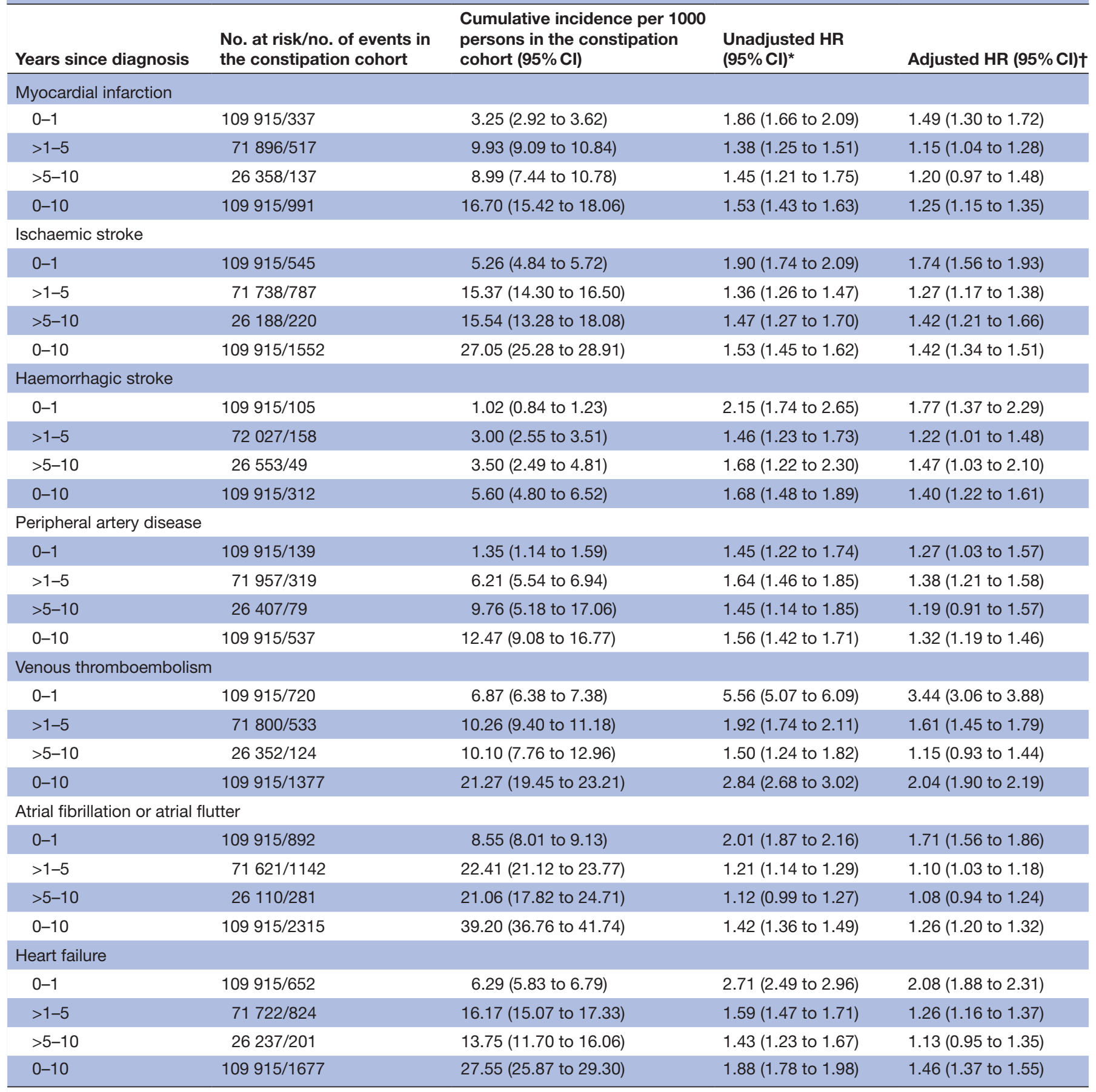

${ }^{*}$ Controlled for matching factors (age, sex, calendar year) by study design.

†Controlled for matching factors (age, sex and calendar year) by study design and adjusted for hypothyroidism, hyperthyroidism, pregnancy within 90 days of the index date, depression, Parkinson's disease, multiple sclerosis, colon, rectal and anal cancer, other gastrointestinal cancers, Crohn's disease, ulcerative colitis, paralytic ileus, chronic pulmonary disease, valvular heart disease, diabetes mellitus, hypertension, hypercholesterolemia, obesity, chronic kidney disease, liver disease, alcoholism-related disorders, medications associated with constipation (iron supplements, opioids, calcium channel blockers, anticholinergic drugs, dopaminergic drugs, tricyclic antidepressants, diuretics, aspirin and non-steroidal anti-inflammatory drugs) and cardiovascular drugs (vitamin $\mathrm{K}$ antagonists, direct oral anticoagulants, angiotensin-converting enzyme inhibitors, angiotensin receptor blockers, beta-blockers, P2Y12 inhibitors, digoxin, amiodarone, nitrates and statins).

higher for unspecified stroke. When we restricted the analysis to stroke diagnoses confirmed by CT or MRI of the brain during the incident hospital contact for stroke, the results remained unchanged (online supplementary table S10). For myocardial infarction (aHR 1.24, 95\% CI 1.14 to 1.35 ), the E-value was 1.79 (E-value for lower limit 
Table 3 Risk of cardiovascular events among patients with constipation relative to the general population cohort, by use of laxatives in the constipation cohort from 1 year before to 3 months after the constipation diagnosis ${ }^{\star}$

\begin{tabular}{|c|c|c|c|c|}
\hline & \multicolumn{2}{|c|}{$\begin{array}{l}\text { Cumulative incidence per } 1000 \text { persons in the } \\
\text { constipation cohort }(95 \% \mathrm{Cl})\end{array}$} & \multicolumn{2}{|c|}{ Adjusted HR $(95 \% \mathrm{Cl}) \dagger$} \\
\hline & $\begin{array}{l}0 \text { to } 1 \text { prescriptions for } \\
\text { laxatives } \\
(n=68698)\end{array}$ & $\begin{array}{l}\geq 2 \text { prescriptions for } \\
\text { laxatives } \\
(n=3125)\end{array}$ & $\begin{array}{l}0-1 \text { prescriptions } \\
\text { for laxatives } \\
(n=68698)\end{array}$ & $\begin{array}{l}\geq 2 \text { prescriptions for } \\
\text { laxatives } \\
(n=3125)\end{array}$ \\
\hline Myocardial infarction & 17.88 (16.20 to 19.69$)$ & 12.78 (7.59 to 20.36$)$ & 1.17 (1.07 to 1.28$)$ & 2.40 (1.05 to 5.49$)$ \\
\hline Ischaemic stroke & 31.08 (28.63 to 33.68$)$ & 19.59 (12.47 to 29.37$)$ & 1.39 (1.30 to 1.49$)$ & 3.22 (1.72 to 6.03$)$ \\
\hline Peripheral artery disease & $16.83(10.82$ to 25.08$)$ & 7.28 (2.34 to 18.29$)$ & 1.38 (1.22 to 1.55$)$ & $0.88(0.20$ to 3.89$)$ \\
\hline Venous thromboembolism & 19.54 (16.99 to 22.35$)$ & 28.15 (19.60 to 39.11$)$ & 1.66 (1.52 to 1.82$)$ & 5.15 (2.63 to 10.08$)$ \\
\hline Atrial fibrillation or atrial flutter & 42.44 (39.00 to 46.08$)$ & 25.93 (18.42 to 35.44$)$ & 1.12 (1.06 to 1.19$)$ & $1.37(0.76$ to 2.45$)$ \\
\hline Heart failure & 29.58 (27.36 to 31.94$)$ & 23.27 (14.67 to 35.10$)$ & 1.36 (1.26 to 1.46$)$ & 1.76 (0.90 to 3.42$)$ \\
\hline
\end{tabular}

The dash (-) indicates insufficient data for an estimate.

${ }^{*}$ The analysis was performed as a landmark analysis, starting follow-up 3 months after the constipation diagnosis. †Controlled for matching factors (age, sex and calendar year) by study design and adjusted for hypothyroidism, hyperthyroidism, pregnancy within 90 days before the index date, depression, Parkinson's disease, multiple sclerosis, colon, rectal and anal cancer, other gastrointestinal cancers, Crohn's disease, ulcerative colitis, paralytic ileus, chronic pulmonary disease, valvular heart disease, diabetes mellitus, hypertension, hypercholesterolemia, obesity, chronic kidney disease, liver disease, alcoholism-related disorders, medications associated with constipation (iron supplements, opioids, calcium channel blockers, anticholinergic drugs, dopaminergic drugs, tricyclic antidepressants, diuretics, aspirin and non-steroidal anti-inflammatory drugs) and cardiovascular drugs (vitamin $\mathrm{K}$ antagonists, direct oral anticoagulants, angiotensinconverting enzyme inhibitors, angiotensin receptor blockers, beta-blockers, P2Y12 inhibitors, digoxin, amiodarone, nitrates and statins). NSAIDs, non-steroidal anti-inflammatory drugs.

of $95 \% \mathrm{CI}=1.54)$. For venous thromboembolism (aHR 2.04, $95 \%$ CI 1.89 to 2.20 ), the E-value was 3.50 (E-value for lower limit of $95 \% \mathrm{CI}=3.19$ ).

\section{DISCUSSION}

In this nationwide, matched, population-based cohort study with a virtually complete follow-up of 83239 patients with constipation, we showed that constipation was associated with an increased risk of all cardiovascular outcomes in the short term and primarily associated with venous thromboembolism and ischaemic stroke beyond 5 years of follow-up. The associations were generally stronger for venous than arterial events and strongest for splanchnic venous thrombosis. The associations were strengthened in patients with more intense use of laxatives, especially for the outcomes ischaemic stroke and venous thromboembolism. When applying a redefined algorithm for our constipation cohort using prescriptions for laxatives in order to capture presumably milder cases of constipation in primary care, our results were similar to the results of the main analysis. We observed an increasing risk of cardiovascular outcomes with decreasing age, presumably due to a low absolute risk of in younger age groups, where the relative effect of constipation is more pronounced.

\section{Comparison with other studies}

Two American studies have focused on constipation as a risk factor for cardiovascular disease, both examining only atherosclerotic cardiovascular disease. ${ }^{24} 25$ In a prospective cohort of 73047 community-dwelling postmenopausal women enrolled in the Women's Health Initiative in the USA, constipation was reported among $35 \%$. After adjusting for confounding factors, only severe constipation remained moderately associated with a composite outcome of atherosclerotic cardiovascular events, with an aHR of 1.23 (95\% CI 1.03 to 1.47$).{ }^{24}$ In contrast, in a cohort of 3359653 US veterans, $7 \%$ reported constipation. Patients with constipation (versus without) had aHRs of 1.11 (95\% CI 1.08 to 1.14) for coronary heart disease and 1.19 (95\% CI 1.15 to 1.22) for ischaemic stroke. Patients taking 1 and $\geq 2$ types of laxatives (versus none) experienced a similarly increased risk. ${ }^{25}$ In both studies, constipation was self-reported, which is less specific than symptom-based criteria. ${ }^{7}$ Therefore, the constipation cases were likely milder than the physician-evaluated, hospital-based cases included in our study. This could explain why patients with self-perceived constipation had only a modestly increased risk of cardiovascular events. Another cohort study examined the association between self-reported bowel movement frequency and risk of cardiovascular disease (coronary heart disease or stroke) among participants in the Nurses' Health Study in the USA. $^{38}$ The study included 86289 women and followed them for up to 30 years. After adjustment, the study found no association with incident cardiovascular disease.

Our study complements the literature by examining patients with physician-diagnosed constipation in a population-based, nationwide setting and comparing with matched individuals from the general population. In contrast to previous studies, we also examined the association with venous thromboembolism. Moreover, we included a wide range of separately analysed 
cardiovascular outcomes, beyond atherosclerotic disease, while retaining the precision of the effect estimates in several subgroup analyses.

\section{Potential mechanisms}

Putative mechanisms through which constipation may increase cardiovascular risk are likely multifactorial and may differ by type of outcome. Increased risk of venous events may be related to shared risk factors, such as physical inactivity and obesity. This could explain the observation of a markedly higher risk of splanchnic venous thrombosis compared with deep venous thrombosis and pulmonary embolism.

Decreased gut motility in patients with constipation may cause dysbiosis of the microbiota. ${ }^{21}$ Recently, the contributory role of the gut microbiota in the development of atherosclerosis and cardiovascular disease has been observed consistently in several large clinical cohorts. ${ }^{18-20}$ The increased transit time in constipated patients may further facilitate the translocation of proinflammatory cytokines from gut bacteria and result in heightened inflammatory responses as well as oxidative stress. ${ }^{21}$ Thus, patients with constipation may sustain a state of systemic low-grade inflammation, ${ }^{21}$ which accelerates the development of atherosclerosis. ${ }^{39}$ Such a mechanism may explain the association between constipation and disease development in distant cardiovascular beds and underlie our observation of an increased risk of myocardial infarction, ischaemic stroke and peripheral arterial disease. In a recent paper, $^{40}$ a positive association between constipation and various gastrointestinal cancers has been established. This may partly explain our observation of a much higher risk of splanchnic venous thrombosis than deep venous thrombosis and pulmonary embolism. Increased blood pressure, a universal cardiovascular risk factor, may also be at the root of the observed association with various cardiovascular diseases. Constipation leads to straining at stool, which has been associated with transient increases in blood pressure. ${ }^{10}$ Moreover, chronic constipation induces psychological stress, ${ }^{41}$ which may increase blood pressure. All of the above mechanisms are presumably related temporally to the peak of constipation symptoms, which likely occurs at time of diagnosis. This may explain our observation of highest cardiovascular risk during the first year after the constipation diagnosis.

We observed an increased risk for all outcomes in patients with more intense use of laxatives, except for peripheral artery disease. This may reflect more severe constipation in these patients; however, confounding by indication may also partly underlie this observation. As an example, patients with severe pain from cancer or ischaemic heart disease may become constipated from morphine use, which in turn may drive the more intense use of laxatives.

Though we analysed each cardiovascular outcome separately, it is likely that relationships exist between them; for example, a first occurrence of myocardial infarction may drive the development of heart failure. Similarly, all cardiovascular outcomes in our study, except heart failure and haemorrhagic stroke, require anticoagulation or antithrombotic therapy, which may mediate the increased risk of haemorrhagic stroke observed in our study.

Reverse causation is possible for outcomes with the potential for slow onset and a long prediagnostic phase, that is, certain cases of heart failure and atrial fibrillation or flutter. Both diseases can lead to dyspnoea and decreased activity, which in turn can induce constipation. ${ }^{13}$ The remaining outcomes of the study are characterised by acute onset, specific symptoms and severe course and are unlikely to precede constipation undiagnosed.

\section{Strengths and weaknesses of the study}

Several strengths and limitations should be considered when interpreting our results. The population-based design within the setting of a tax-supported universal healthcare system with complete follow-up of all patients largely eliminated selection bias stemming from selective inclusion of specific hospitals, health insurance systems or income levels. ${ }^{27}$ The positive predictive value of the DNPR data is high for diagnoses of myocardial infarction $(97 \%)$, ischaemic stroke (97\%), haemorrhagic stroke $(88 \%)$, peripheral artery disease $(91 \%)$, venous thromboembolism $(88 \%)$ and atrial fibrillation or atrial flutter $(95 \%)$, but somewhat lower for heart failure $(80 \%) .{ }^{28} 3342$ Constipation diagnoses in the DNPR are based on an evaluation by the treating physician. Therefore, the positive predictive value is assumed to be high, whereas sensitivity and other aspects of validity may be lower.

Surveillance and detection bias cannot be excluded. However, most outcomes included in the study are characterised by acute onset and a severe clinical course, making them less susceptible to surveillance bias. Furthermore, we observed no compensatory deficit in estimates for any outcome during $>1-5$ years of follow-up. Such deficits would be expected if detection bias was present during the first year of follow-up.

We used several approaches to control for and examine the potential impact of confounding. We adjusted our analyses for a wide range of conditions and drugs associated with constipation, as well as cardiovascular and life style diseases and associated drugs. Our results remained largely unchanged in analyses restricted to patients with no cardiovascular risk factors at baseline, indicating that confounding by cardiovascular comorbidity may not be prominent. However, a degree of unmeasured confounding may be expected by physical inactivity and dietary habits, which are risk factors for both constipation and cardiovascular disease. ${ }^{13}$ Moreover, obesity was incompletely captured $(4.0 \%$ in the constipation cohort and $2.1 \%$ in the comparison cohort), and residual confounding by obesity is possible despite adjusting for associated lifestyle conditions. The derived E-values indicating the strength of association with both the exposure and the outcome needed by an unmeasured confounder to potentially (as a maximum) explain away selected representative findings for myocardial infarction and 
venous thromboembolism (aHR 1.24 and 2.04) were relative large (E-values 1.79 and 3.50). For example, the association between obesity and constipation is 1.10 (95\% CI 0.69 to 1.75$) ;{ }^{43}$ therefore, residual confounding by obesity is unlikely to be an important confounder. However, multiple unmeasured confounders may in concert explain our results to an unknown degree.

\section{Conclusion}

Constipation was associated with an increased risk of the most common cardiovascular diseases. The strongest associations were observed for venous thromboembolism.

Contributors JS, KA, HG and HTTS conceived the study idea and designed the study. JS directed the analyses, which were carried out by SKS and PS. All authors participated in the discussion and interpretation of the results. JS reviewed the literature, organised the writing and wrote the initial drafts. All authors critically revised the manuscript for intellectual content and approved the final version. HTTS is the guarantor.

Funding The authors have not declared a specific grant for this research from any funding agency in the public, commercial or not-for-profit sectors. This work was supported by the Danish Cancer Society (grant no. R73-A4284-13-S17) and the Aarhus Research Foundation. All authors had full access to the study data and can take responsibility for the integrity of the data and the accuracy of the data analysis and had final responsibility for the decision to submit for publication.

Disclaimer The sponsors had no role in study design, data collection, analysis or interpretation of the data, or writing of the manuscript or in the decision to submit the paper for publication.

Competing interests None declared.

Patient consent for publication Not required.

Ethics approval The study was approved by the Danish Data Protection Agency (record number: 2006-53-1396). Informed consent and approval from an ethics committee are not required for registry-based studies according to Danish legislation.

Provenance and peer review Not commissioned; externally peer reviewed.

Data availability statement All data relevant to the study are included in the article or uploaded as supplementary information. № additional data are available.

Open access This is an open access article distributed in accordance with the Creative Commons Attribution Non Commercial (CC BY-NC 4.0) license, which permits others to distribute, remix, adapt, build upon this work non-commercially, and license their derivative works on different terms, provided the original work is properly cited, appropriate credit is given, any changes made indicated, and the use is non-commercial. See: http://creativecommons.org/licenses/by-nc/4.0/.

\section{ORCID iDs}

Jens Sundbøll http://orcid.org/0000-0002-1276-1419

Kasper Adelborg http://orcid.org/0000-0001-5639-7252

\section{REFERENCES}

1 Wright PS, Thomas SL. Constipation and diarrhea: the neglected symptoms. Semin Oncol Nurs 1995;11:289-97.

2 Peppas G, Alexiou VG, Mourtzoukou E, et al. Epidemiology of constipation in Europe and Oceania: a systematic review. BMC Gastroenterol 2008;8:5.

3 Peery AF, Crockett SD, Barritt AS, et al. Burden of gastrointestinal, liver, and pancreatic diseases in the United States. Gastroenterology 2015:149:1731-41.

4 Higgins PDR, Johanson JF. Epidemiology of constipation in North America: a systematic review. Am J Gastroenterol 2004;99:750-9.

5 Mugie SM, Benninga MA, Di Lorenzo C. Epidemiology of constipation in children and adults: a systematic review. Best Pract Res Clin Gastroenterol 2011;25:3-18.

6 Pare P, Ferrazzi S, Thompson WG, et al. An epidemiological survey of constipation in Canada: definitions, rates, demographics, and predictors of health care seeking. Am J Gastroentero 2001;96:3130-7.
7 Stewart WF, Liberman JN, Sandler RS, et al. Epidemiology of constipation (EPOC) study in the United States: relation of clinical subtypes to sociodemographic features. Am J Gastroenterol 1999;94:3530-40.

8 Drossman DA, Li Z, Andruzzi E, et al. U.S. householder survey of functional gastrointestinal disorders. Prevalence, sociodemography, and health impact. Dig Dis Sci 1993;38:1569-80.

9 Talley NJ, Weaver AL, Zinsmeister AR, et al. Functional constipation and outlet delay: a population-based study. Gastroenterology 1993;105:781-90.

10 Ishiyama $\mathrm{Y}$, Hoshide S, Mizuno H, et al. Constipation-induced pressor effects as triggers for cardiovascular events. J Clin Hypertens 2019;21:421-5.

11 Chang JY, Locke GR, Schleck CD, et al. Risk factors for chronic constipation and a possible role of analgesics. Neurogastroenterol Motil 2007;19:905-11.

12 Camilleri M, Ford AC, Mawe GM, et al. Chronic constipation. Nat Rev Dis Primers 2017;3:17095.

13 Dukas L, Willett WC, Giovannucci EL. Association between physical activity, fiber intake, and other lifestyle variables and constipation in a study of women. Am J Gastroenterol 2003;98:1790-6.

14 Vilahur G, Badimon JJ, Bugiardini R, et al. Perspectives: the burden of cardiovascular risk factors and coronary heart disease in Europe and worldwide. European Heart Journal Supplements 2014;16:A7-11.

15 Mancabelli L, Milani C, Lugli GA, et al. Unveiling the gut microbiota composition and functionality associated with constipation through metagenomic analyses. Sci Rep 2017;7:9879.

16 Menni C, Lin C, Cecelja M, et al. Gut microbial diversity is associated with lower arterial stiffness in women. Eur Heart J 2018;39:2390-7.

17 Yang T, Santisteban MM, Rodriguez V, et al. Gut dysbiosis is linked to hypertension. Hypertension 2015;65:1331-40.

18 Tang WHW, Wang Z, Levison BS, et al. Intestinal microbial metabolism of phosphatidylcholine and cardiovascular risk. N Engl $J$ Med 2013;368:1575-84.

19 Tang WHW, Hazen SL. The contributory role of gut microbiota in cardiovascular disease. J Clin Invest 2014;124:4204-11.

20 Wang Z, Klipfell E, Bennett BJ, et al. Gut flora metabolism of phosphatidylcholine promotes cardiovascular disease. Nature 2011;472:57-63.

21 Tang WHW, DY L, Hazen SL, et al. The gut microbiome, and heart failure. Nat Rev Cardiol 2019;16:137-54.

22 Rapsomaniki E, Timmis A, George J, et al. Blood pressure and incidence of twelve cardiovascular diseases: lifetime risks, healthy life-years lost, and age-specific associations in 1.25 million people. Lancet 2014;383:1899-911.

23 Benjamin EJ, Levy D, Vaziri SM, et al. Independent risk factors for atrial fibrillation in a population-based cohort. The Framingham heart study. JAMA 1994;271:840-4.

24 Salmoirago-Blotcher E, Crawford S, Jackson E, et al. Constipation and risk of cardiovascular disease among postmenopausal women. Am J Med 2011;124:714-23.

25 Sumida K, Molnar MZ, Potukuchi PK, et al. Constipation and risk of death and cardiovascular events. Atherosclerosis 2019;281:114-20.

26 Schmidt M, Schmidt SAJ, Adelborg K, et al. The Danish health care system and epidemiological research: from health care contacts to database records. Clin Epidemiol 2019;11:563-91.

27 Schmidt M, Pedersen L, Sørensen HT. The Danish civil registration system as a tool in epidemiology. Eur J Epidemiol 2014;29:541-9.

28 Schmidt M, Schmidt SAJ, Sandegaard JL, et al. The Danish national patient registry: a review of content, data quality, and research potential. Clin Epidemiol 2015;7:449-90.

29 Johannesdottir SA, Horváth-Puhó E, Ehrenstein V, et al. Existing data sources for clinical epidemiology: the Danish national database of reimbursed prescriptions. Clin Epidemiol 2012;4:303-13.

30 Lund JL, Horváth-Puhó E, Komjáthiné Szépligeti S, et al. Conditioning on future exposure to define study cohorts can induce bias: the case of low-dose acetylsalicylic acid and risk of major bleeding. Clin Epidemiol 2017;9:611-26.

31 Heide-Jørgensen U, Adelborg K, Kahlert J, et al. Sampling strategies for selecting general population comparison cohorts. Clin Epidemiol 2018;10:1325-37.

32 Sørensen HT. Venous thromboembolism and the concepts of the incidence and mortality. J Thromb Haemost 2007;5:690-1.

33 Sundbøll J, Adelborg K, Munch T, et al. Positive predictive value of cardiovascular diagnoses in the Danish national patient registry: a validation study. BMJ Open 2016;6:e012832.

34 Krarup L-H, Boysen G, Janjua $\mathrm{H}$, et al. Validity of stroke diagnoses in a national register of patients. Neuroepidemiology 2007;28:150-4.

35 Satagopan JM, Ben-Porat L, Berwick M, et al. A note on competing risks in survival data analysis. Br J Cancer 2004;91:1229-35. 
36 Therneau T. Modeling survival data: extending the COX model. Berlin, Germany: Springer Science \& Business Media, 2000: 44-8.

37 VanderWeele TJ, Ding P. Sensitivity analysis in observational research: introducing the E-Value. Ann Intern Med 2017;167:268-74.

$38 \mathrm{Ma} \mathrm{W}, \mathrm{Li}$ Y, Heianza Y, et al. Associations of bowel movement frequency with risk of cardiovascular disease and mortality among US women. Sci Rep 2016;6:33005.

39 Libby P, Ridker PM, Maseri A. Inflammation and atherosclerosis. Circulation 2002;105:1135-43.
40 Sundbøll J, Thygesen SK, Veres K, et al. Risk of cancer in patients with constipation. Clin Epidemiol 2019;11:299-310.

41 Merkel IS, Locher J, Burgio K, et al. Physiologic and psychologic characteristics of an elderly population with chronic constipation. Am $J$ Gastroenterol 1993;88:1854-9.

42 Hald SM, Kring Sloth C, Hey SM, et al. Intracerebral hemorrhage: positive predictive value of diagnosis codes in two nationwide Danish registries. Clin Epidemiol 2018;10:941-8.

43 Costa ML, Oliveira JN, Tahan S, et al. Overweight and constipation in adolescents. BMC Gastroenterol 2011;11:40. 